\title{
DNA methylation-induced E-cadherin silencing is correlated with the clinicopathological features of melanoma
}

\author{
MARIO VENZA ${ }^{1 *}$, MARIA VISALLI $^{1 *}$, TERESA CATALANO $^{1}$, CARMELO BIONDO $^{2}$, \\ CONCETTA BENINATI $^{2,3}$, DIANA TETI ${ }^{1}$ and ISABELLA VENZA ${ }^{1}$
}

\begin{abstract}
Departments of ${ }^{1}$ Clinical and Experimental Medicine and ${ }^{2}$ Human Pathology of Adult and Developmental Age 'Gaetano Barresi', Azienda Policlinico Universitario G. Martino, ${ }^{3}$ Scylla Biotech Srl, University of Messina, Messina, Italy
\end{abstract}

Received November 6, 2015; Accepted January 22, 2016

DOI: $10.3892 /$ or.2016.4618

\begin{abstract}
E-cadherin, a calcium-dependent cell-cell adhesion molecule, has an important role in epithelial cell function, maintenance of tissue architecture and cancer suppression. Loss of E-cadherin promotes tumor metastatic dissemination and predicts poor prognosis. The present study investigated the clinicopathological significance of E-cadherin expression in cutaneous, mucosal and uveal melanoma related to epigenetic mechanisms that may contribute to $E$-cadherin silencing. E-cadherin expression was reduced in 55/130 cutaneous (42.3\%), 49/82 mucosal (59.7\%) and 36/64 uveal (56.2\%) melanoma samples as compared to normal skin controls and was inversely associated with promoter methylation. Of the 10 different CpG sites studied (nt 863, 865, 873, 879, 887, 892, 901, 918, 920 and 940), two sites (nt 892 and 940) were 90-100\% methylated in all the melanoma specimens examined and the other ones were partially methylated (range, 53-86\%). In contrast, the methylation rate of the E-cadherin gene was low in normal tissues (range, 5-24\%). In all the three types of melanoma studied, a significant correlation was found between reduced levels of E-cadherin and reduced survival, high mitotic index and metastasis, accounting for the predilection of lymph nodal localization. In cutaneous and mucosal melanoma, low E-cadherin expression was positively correlated also with head/neck localization and ulceration. A high
\end{abstract}

Correspondence to: Professor Diana Teti, Department of Clinical and Experimental Medicine, Azienda Policlinico Universitario G. Martino, via Consolare Valeria 1, I-98125 Messina, Italy

E-mail: dteti@unime.it

"Contributed equally

Abbreviations: FFPE, formalin-fixed paraffin-embedded; NHEMs, normal human epidermal melanocytes; qPCR, quantitative real-time PCR; MSP, methylation-specific PCR; HPF, high-power field; TSS, transcriptional start site

Key words: E-cadherin, gene expression, DNA methylation, clinicopathological parameters, melanoma frequency of reduced E-cadherin levels occurred in choroid melanomas. In vitro experiments showed that E-cadherin transcription was restored following 5-aza-2'-deoxycytidine (5-aza-dC) treatment or DNMT1 silencing and was negatively correlated with the invasive potential of melanoma cells. The significant relationship between E-cadherin silencing and several poor prognostic factors indicates that this adhesion molecule may play an important role in melanomagenesis. Therefore, the inverse association of E-cadherin expression with promoter methylation raises the intriguing possibility that reactivation of E-cadherin expression through promoter demethylation may represent a potential therapeutic strategy for the treatment of melanoma.

\section{Introduction}

In recent years, the incidence and mortality rate of melanoma have considerably increased (1), whereby the need to discover new therapeutic strategies has become increasingly urgent (2-6). E-cadherin has been shown to play a key role in cell adhesion, growth and differentiation (7), frequently overexpressed in melanoma. Several studies have revealed that E-cadherin is expressed in the normal epidermis and lost in invasive and metastatic melanoma cells (8). Depletion of functional E-cadherin induces the escape of melanocytes from keratinocyte-mediated growth and phenotypic control, thus allowing invasion and migration (9).

Although reduced expression of E-cadherin is known to be caused by promoter hypermethylation in several types of tumors (10), such an epigenetic event has been only partially investigated in melanoma. Moreover, the existence of a relationship between epigenetic changes, E-cadherin expression and clinicopathological features has not yet been evaluated in melanoma.

In the present study, we aimed to investigate whether E-cadherin expression is epigenetically regulated in a cohort of patients affected by cutaneous, mucosal and uveal melanoma and is associated with specific clinicopathological parameters. Findings from the present study revealed a strong association between E-cadherin promoter methylation and several significant pathological characteristics, whereby they may provide insights into the biology of this cancer. 


\section{Materials and methods}

Cell cultures. Normal human epidermal melanocyte (NHEM) cells were grown in Melanocyte Medium plus Bullet kit (both from Lonza, Walkersville, MD, USA). Cutaneous (G361, WM-115 and WM-266-4), mucosal (MMel-1 and MMel-2) and uveal (OCM-1, OCM-3 and 92.1) melanoma cells were grown as previously described $(11,12)$.

Treatment with the DNA demethylating agent 5-aza-dC. Cells were treated with 5-aza-2'-deoxycytidine (5-aza-dC) (Sigma Chemical Co.) by addition of fresh medium containing 5-aza-dC $(10 \mu \mathrm{mol} / \mathrm{l})$ every day for three consecutive days.

Tissue specimens. Formalin-fixed paraffin-embedded (FFPE) tissue sections of 130 cutaneous, 82 mucosal and 64 uveal melanomas, and 65 normal skin specimens were collected from the Department of Human Pathology, University of Messina, Messina, Italy. The data regarding patients are shown in Table I. The investigation adhered to the Declaration of Helsinki and was approved by the Ethics Committee of the University Hospital of Messina. Informed consent was provided by the patients.

Determination of mitotic index. Mitotic index was determined by counting the number of mitoses in 10 consecutive nonoverlapping high power fields (HPFs) with commencement in an area of high mitotic activity using a magnification of $x 400$.

DNA and RNA extraction. Total RNA and DNA extraction from cells was performed using TRIzol reagent (Invitrogen), and Recover All Total Nucleic Acid Isolation kit (Ambion Inc., Austin, TX, USA) was used for extractin from FFPE samples.

Reverse transcription and $q P C R$. Total RNA was reverse-transcribed with IMProm-II ${ }^{\mathrm{TM}}$ Reverse Transcriptase kit (Promega, Milan, Italy). qPCR was performed using the ABI Prism 7500 Real-Time PCR system (Applied Biosystems, Milan, Italy). Primers and probes were previously described (11). The mRNA levels of E-cadherin were normalized to endogenous $\beta$-actin (Applied Biosystems). The control was represented by NHEM cells (described in the paragraph 'Cell culture'). Their expression was considered as 1 .

Bisulfite modification and MSP. Bisulfite-modified DNA obtained using the EpiTect Bisulfite kit (Qiagen, Milan, Italy) was amplified using the following primers as previously reported (13): methylated DNA-specific primers: forward primer, 5'-TTAGGTTAGAGGGTTATCGCGT-3' and reverse primer, 5'-TAACTAAAAATTCACCTACCGAC-3' (115 bp; genomic position relative to TSS, -176/-61); unmethylated DNA-specific primers: forward primer, 5'-TAATTTTAGGTT AGAGGGTTATTGT-3' and reverse primer, 5'-CACAACCAA TCAACAACACA-3' (97 bp; genomic position relative to TSS, -181/-84). PCR products were separated by $2 \%$ agarose gel containing ethidium bromide.

Bisulfite genomic sequencing. Bisulfite-treated DNA was amplified by PCR with primers that were specific for modified DNA but did not contain any $\mathrm{CpG}$ sites in their sequence.
Table I. Patient characteristics.

\begin{tabular}{|c|c|c|c|}
\hline Characteristics & $\begin{array}{c}\text { Cutaneous } \\
\text { melanoma } \\
(\mathrm{n}=130) \\
\mathrm{n}(\%)\end{array}$ & $\begin{array}{c}\text { Mucosal } \\
\text { melanoma } \\
(\mathrm{n}=82) \\
\mathrm{n}(\%)\end{array}$ & $\begin{array}{c}\text { Uveal } \\
\text { melanoma } \\
(\mathrm{n}=64) \\
\mathrm{n}(\%)\end{array}$ \\
\hline Males & $78(60)$ & $40(48.8)$ & $31(48.4)$ \\
\hline Females & $52(40)$ & $42(51.2)$ & $33(51.6)$ \\
\hline \multicolumn{4}{|l|}{ AJCC stage } \\
\hline $\mathrm{I}$ & $34(26.2)$ & $23(28.0)$ & $14(21.9)$ \\
\hline II & $38(29.2)$ & $19(23.2)$ & $14(21.9)$ \\
\hline III & $30(23.1)$ & $24(29.3)$ & $18(28.1)$ \\
\hline IV & $28(21.5)$ & $16(19.5)$ & $18(28.1)$ \\
\hline \multicolumn{4}{|l|}{ Site } \\
\hline Extremities & $39(30)$ & & \\
\hline Trunk & $26(20)$ & & \\
\hline Head/neck & $65(50)$ & & \\
\hline Vulvovaginal & & $28(34.1)$ & \\
\hline Anorectal & & $22(26.9)$ & \\
\hline Head/neck & & $32(39.0)$ & \\
\hline Choroid & & & $34(53.1)$ \\
\hline Ciliary body & & & $17(26.6)$ \\
\hline Iris & & & $13(20.3)$ \\
\hline \multicolumn{4}{|l|}{ Ulceration } \\
\hline Absent & $83(63.8)$ & $34(41.5)$ & $64(100)$ \\
\hline Present & $47(36.2)$ & $48(58.5)$ & $0(0)$ \\
\hline \multicolumn{4}{|c|}{ Breslow thickness (mm) } \\
\hline$\leq 1.00$ & $41(31.5)$ & $23(28.0)$ & $24(37.5)$ \\
\hline $1.01-4.00$ & $42(32.4)$ & $25(30.5)$ & $19(29.7)$ \\
\hline$>4.01$ & $47(36.1)$ & $34(41.5)$ & $21(32.8)$ \\
\hline \multicolumn{4}{|c|}{ Mitotic index (per 10 HPFs) } \\
\hline 1-5 mitoses & $29(22.3)$ & $19(23.2)$ & $12(18.8)$ \\
\hline 6-10 mitoses & $43(33.1)$ & $15(18.3)$ & $18(28.1)$ \\
\hline $11-15$ mitoses & $19(14.6)$ & $18(21.9)$ & $15(23.4)$ \\
\hline $16-20$ mitoses & $39(30.0)$ & $30(36.6)$ & $19(29.7)$ \\
\hline \multicolumn{4}{|c|}{ Metastatic lesions } \\
\hline Present & $64(49.2)$ & $47(57.3)$ & $37(57.8)$ \\
\hline Absent & $66(50.8)$ & $35(42.7)$ & $27(42.2)$ \\
\hline
\end{tabular}

AJCC, American Joint Committee on Cancer; HPFs, high power fields.

The primers were: S1 (TTT AGT AAT TTT AGG TTA GAG GGT T, upstream, nt 836-861; GeneBank accession no. L34545) and S2 (CTA ATT AAC TAA AAA TTC ACC TAC C, downstream, sequence position nt 965-940) (14). The PCR conditions were $94^{\circ} \mathrm{C}$ for $2 \mathrm{~min} ; 35$ cycles of $94^{\circ} \mathrm{C}$ for $20 \mathrm{sec}, 48^{\circ} \mathrm{C}$ for $20 \mathrm{sec}$ and $72^{\circ} \mathrm{C}$ for $30 \mathrm{sec}$; and a final extension at $72^{\circ} \mathrm{C}$ for $5 \mathrm{~min}$. The PCR product was extracted from the gel with the QIAquick Gel Extraction kit (Qiagen). The purified DNA samples were sequenced with the CEQ DTCS Quick Start kit, and with an automated DNA sequencer (Beckman Coulter CEQ 2000 analysis system) (both from Beckman Coulter, S.p.A.). 

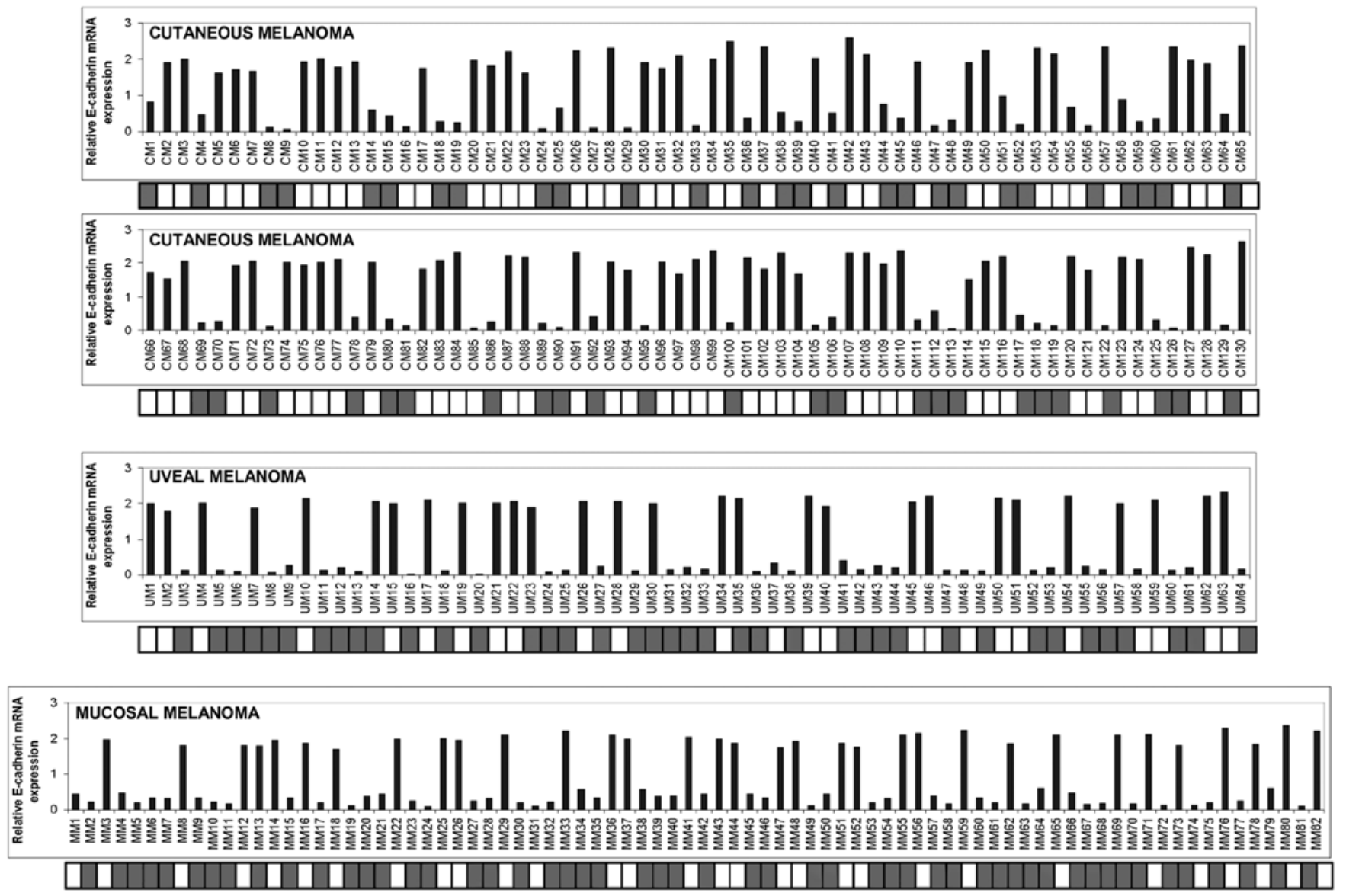

Figure 1. Expression levels and the methylation status of E-cadherin in cutaneous, uveal and mucosal melanoma specimens. (A) Total RNA was extracted from 130 cutaneous melanomas (CM), 64 uveal melanomas and (UM) 82 mucosal melanomas (MM), reverse-transcribed, and analyzed by qPCR. mRNA levels of E-cadherin were normalized using the housekeeping gene $\beta$-actin as the inner control. Data are depicted as the mean \pm SD of three independent experiments. The detailed methylation pattern of E-cadherin as determined by methylation specific-PCR (MSP) was placed and aligned just beneath the expression graphs. White square, unmethylated $\mathrm{CpG}$ regions; grey square, methylated $\mathrm{CpG}$ regions.

Quantification of methylation levels. A single cytosine signal at the corresponding $\mathrm{CpG}$ site was considered $100 \%$ methylation, a single thymine signal was considered no methylation, and overlapping cytosine plus thymine signals were considered partial methylation. In the latter condition, the percentage of methylation was expressed as the ratio of the peak values of the cytosine to cytosine plus thymine signals.

Transient transfections. For transient knockdown of DNMT1, $D N M T 3 a$ and DNMT3b, cells were transfected with specific targeting small interfering RNA (siRNA) or non-targeting control siRNA (Invitrogen, Milan, Italy) at a final concentration of $100 \mathrm{nM} 24 \mathrm{~h}$ after plating using siPORT Lipid Transfection Agent (Ambion, Milan, Italy).

Invasion assay. The anti-invasive activity of 5-aza-dC was assessed using the Cultrex ${ }^{\circledR}$ BME Cell Invasion assay (Trevigen, Gaithersburg, MD, USA).

Statistical analysis. The Pearson's correlation test was used to assess the association of E-cadherin expression with promoter methylation. Differences in E-cadherin expression levels and clinical characteristics were evaluated by $\chi^{2}$ test between patient subgroups. Kaplan-Meier method was used in the evaluation of the overall and disease-free survival time. The log-rank test was used in comparing the differences between the periods of survival among the examined patients. A p-value of $<0.05$ was considered to indicate a statistically significant result.

\section{Results}

E-cadherin expression correlates with promoter methylation in cutaneous, uveal and mucosal melanoma. E-cadherin mRNA values were firstly measured in 65 normal skin samples from healthy donors and in NHEM cells in order to determine the cut-off point for abnormal E-cadherin expression. E-cadherin levels (as defined by the ratio between the values measured in skin samples over those of NHEM cells) ranged between 1.52 and 2.1 (mean, 1.84 \pm 0.133 ). Values equal or below 1.44 (determined as the mean minus $3 \mathrm{SD}$ ) were considered to represent under-expression of E-cadherin. As shown in Fig. 1, a strong reduction or loss of expression of E-cadherin was detected in 55/130 cutaneous (42.3\%), 36/64 uveal (56.2\%) and 49/82 mucosal (59.7\%) melanoma samples. In order to investigate whether promoter hypermethylation correlated with $E$-cadherin expression, we performed MSP analysis in the series of melanoma specimens examined. Of the 55 cutaneous melanomas down-expressing E-cadherin, 51 samples were 


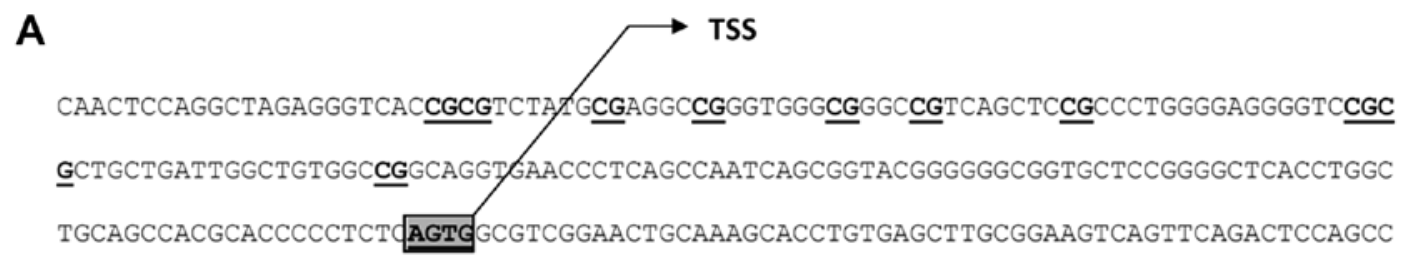
CGCTCCAGCCCGGCCCGACCCGACCGCACCCGGCGCCTGCCCTCGCTCGGCGTCCCCGGCCAGCCATGGGCCCTTGGA

B

$\square$ normal skin $\square$ cutaneous melanoma

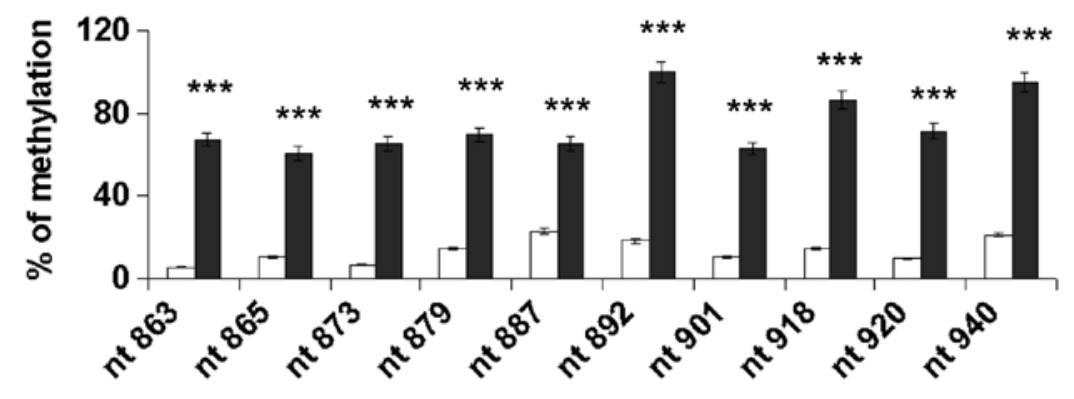

$\square$ normal skin $\square$ uveal melanoma

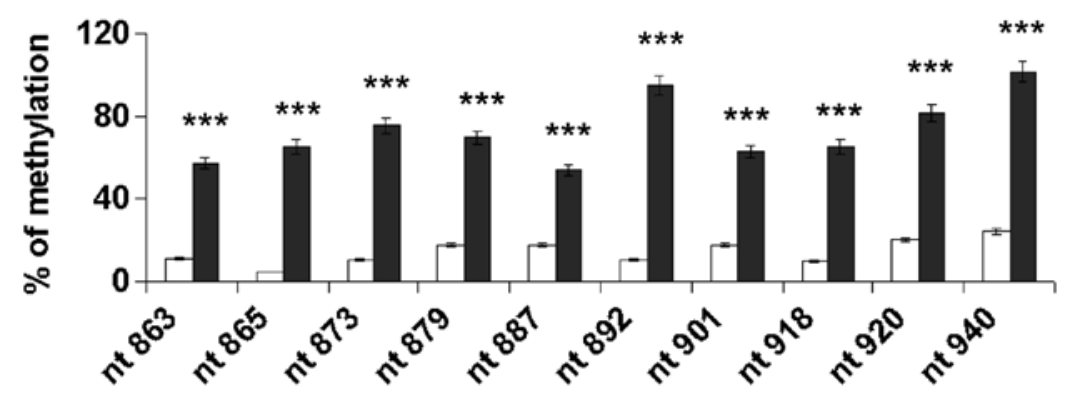

normal skin mucosal melanoma

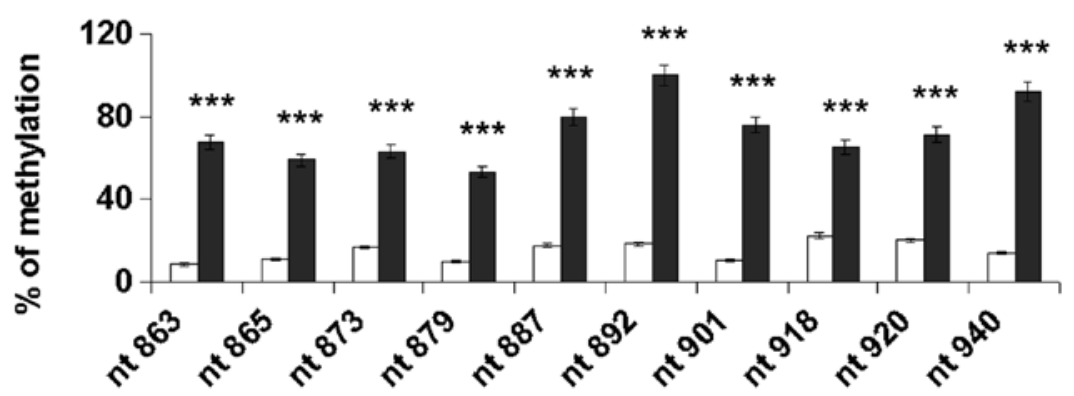

Figure 2. Methylation status of individual CpG sites in the melanoma samples. (A) Schematic representation of the E-cadherin promoter region analyzed. Individual $\mathrm{CpG}$ sites are underlined. (B) Direct bisulfite sequencing analysis of E-cadherin promoter methylation in melanoma. PCR products from normal skin and cutaneous, uveal and mucosal melanoma samples were sequenced after amplification with primers S1/S2, as described in the Materials and methods section. Data are depicted as the mean \pm SD of three independent experiments. Significant ${ }^{* * *}$ p $<0.001$, melanoma samples vs. normal skin.

hypermethylated $(92.7 \%)$ and 4 samples were unmethylated (7.3\%). All of the 75 cutaneous melanoma sections which expressed amounts of E-cadherin mRNA above the cut-off point were unmethylated. Of the 36 uveal melanoma samples exhibiting levels of mRNA under the cut-off point, 33 (91.7\%) were methylated and $3(8.3 \%)$ were unmethylated, whereas only 6 out of $28(21.4 \%)$ specimens exhibiting consistent amounts of E-cadherin expression were methylated. Among the 49 mucosal melanomas that expressed lower mRNA levels of E-cadherin, 46 specimens (93.9\%) exhibited promoter methylation and 3 specimens $(6.1 \%)$ were unmethylated.
Six out of $33(18.2 \%)$ mucosal melanoma samples with high levels of E-cadherin were found to be methylated while the rest were unmethylated. Pearson correlation analysis revealed that E-cadherin mRNA levels were inversely correlated with promoter methylation (for cutaneous melanoma, correlation coefficient, $-0.899, \mathrm{p}<0.001$; for uveal melanoma, correlation coefficient, $-0.729, \mathrm{p}<0.001$; for mucosal melanoma, correlation coefficient, $-0.754, \mathrm{p}<0.001)$.

Sodium bisulfite DNA sequencing was carried out to assess the promoter methylation pattern for 10 specific $\mathrm{CpG}$ sites (genomic positions nt 863, 865, 873, 879, 887, 892, 901, 


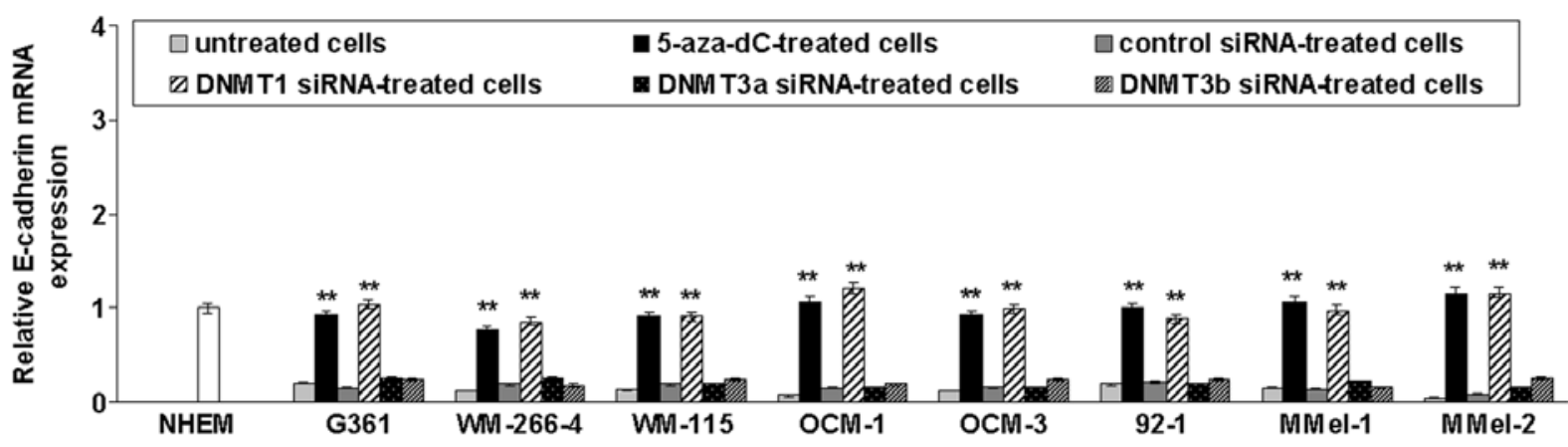

Figure 3. Restoration of E-cadherin gene expression in melanoma cell lines after demethylation. Three cutaneous (G361, WM-266-4 and WM-155), three uveal (OCM-1, OCM-3a and 92.1), and two mucosal (MMel-1 and MMel-2) melanoma cell lines were plated in 6-well plates and exposed to 5-aza-dC $(10 \mu \mathrm{M})$. Where indicated, cells were transfected with a control siRNA or siRNAs targeting DNMT1, DNMT3a and DNMT3b. Total RNA was isolated, reverse-transcribed and analyzed by qRT-PCR. mRNA levels of E-cadherin were normalized using the housekeeping gene $\beta$-actin as the inner control. Data are depicted as the mean \pm SD of three independent experiments. Significant ${ }^{* *} \mathrm{p}<0.01,5$-aza-dC-treated or DNMT1-silenced cells vs. untreated control cells.

918, 920 and 940; GeneBank accession no. L34545), as represented in Fig. 2A. Fig. 2B shows the percentage of methylation of E-cadherin in the normal skin and melanoma samples. Cancer samples showed significantly higher methylation levels compared with the normal tissues $(\mathrm{p}<0.001)$. Notably, the $\mathrm{CpG}$ sites nt 892 and 940 were 90-100\% methylated in all the melanoma types examined and the others were partially methylated (range, 53-86\%). The methylation rate of the E-cadherin gene was low in the normal tissues (range, 5-24\%).

To test whether promoter methylation was responsible for repression of $E$-cadherin transcription, cutaneous (G361, WM-266-4 and WM-155) and uveal (OCM-1, OCM-3 and 92.1) melanoma cell lines previously found to be E-cadherin-methylated (11) as well as primary mucosal melanoma cells (M-Mel-1 and M-Mel-2) that are methylated for this gene (data not shown), were treated with the demethylating drug 5-aza-dC or transfected with siRNAs targeting DNMT1, DNMT3a or DNMT3b. As shown in Fig. 3, E-cadherin mRNA levels were significantly increased following 5-aza-dC treatment or DNMT1 silencing. In contrast, DNMT3a or DNMT3b knockdown did not restore E-cadherin expression.

Correlation between E-cadherin expression and clinical parameters in cutaneous, mucosal and uveal melanoma. We next examined the correlation between E-cadherin expression and different clinicopathological features. Low E-cadherin expression was observed in $78.5 \%$ of the head and neck cutaneous melanomas as compared to $12.8 \%$ of melanomas occurring in other regions $\left(\mathrm{p}<0.0001 ; \chi^{2}\right.$ test $)$. Ulcerated lesions exhibited reduced levels of E-cadherin in $95.7 \%$ of the cases relative to $12.1 \%$ observed in the non-ulcerated tumors $\left(\mathrm{p}<0.0001 ; \chi^{2}\right.$ test). A high percentage of melanomas with elevated mitotic index $(84.2 \%$ for $11-15$ mitoses $/ 10 \mathrm{HPFs}$; $92.3 \%$ for $15-20$ mitoses/10 HPFs) or with metastasis $(68.75 \%)$ showed decreased amounts of E-cadherin as compared with melanomas with a low number of mitoses $(3.5 \%$ for 1-5 mitoses/10 HPFs; $4.7 \%$ for 6-10 mitoses/10 HPFs) $\left(\mathrm{p}<0.0001 ; \chi^{2}\right.$ test $)$, or no metastatic melanomas $(12.1 \%$; $\mathrm{p}<0.0001 ; \chi^{2}$ test), respectively. No statistical differences were found between E-cadherin expression and clinical stage or Breslow thickness (Fig. 4A). Mucosal melanoma presented a similar pattern, since a very significant correlation was found between decreased E-cadherin expression and head/neck localization ( $\mathrm{p}<0.0001 ; \chi^{2}$ test), ulceration ( $\mathrm{p}<0.0001 ; \chi^{2}$ test), mitotic index $\left(\mathrm{p}<0.0001 ; \chi^{2}\right.$ test $)$ and metastasis $(\mathrm{p}<0.0001$; $\chi^{2}$ test) (Fig. 4B). Data relative to uveal melanoma showed that a high frequency of reduced E-cadherin levels occurred in melanomas of the choroid $\left(\mathrm{p}<0.0001 ; \chi^{2}\right.$ test) as well as in those with elevated mitotic index $\left(\mathrm{p}=0.002 ; \chi^{2}\right.$ test $)$ or metastasis $\left(p<0.0001 ; \chi^{2}\right.$ test $)$ (Fig. 4C).

E-cadherin expression discriminates site-specific melanoma metastasis. Analysis of the expression of E-cadherin in metastatic tumor according to the metastasis site revealed that the reduction in E-cadherin expression was significantly associated with lymph node metastatic localization (Fig. 5).

Restoration of E-cadherin negatively correlates with the invasive potential of melanoma cells. Since a relationship between $E$-cadherin silencing and melanoma metastasis was noted (Fig. 4), we aimed at evaluating the effect of 5-aza-dC-induced $E$-cadherin reactivation on melanoma cell invasiveness. As shown in Fig. 6, demethylation markedly inhibited the invasion of all the examined melanoma cell lines.

Correlation between E-cadherin levels and patient survival. The overall and disease-free survival of melanoma patients were also explored and the association with E-cadherin expression was analyzed. As shown in Fig. 7, a shorter survival time for patients with reduced levels of E-cadherin was documented $(\mathrm{p}<0.0001)$. Compared to the overall survival time for patients with preserved levels of E-cadherin (from 2 to 60 months, average 32.3 months, median 36 months in cutaneous melanoma; from 2 months to 60 months, average 33.2 months, median 36 months in mucosal melanoma; from 2 to 60 months, average 25.5 months, median 24 months in uveal melanoma), a shorter overall survival time in patients with reduced expression of E-cadherin was observed (from 2 to 52 months, average 19.4 months, median 20 months in cutaneous melanoma; from 2 to 44 months, average 19.2 months, median 24 months in mucosal melanoma; from 2 to 28 months, average 15.2 months, median 14 months in uveal melanoma). Disease-free survival time was also shorter in patients with loss of expression of E-cadherin, compared with the other patients. In the presence 
A

CUTANEOUS MELANOMA
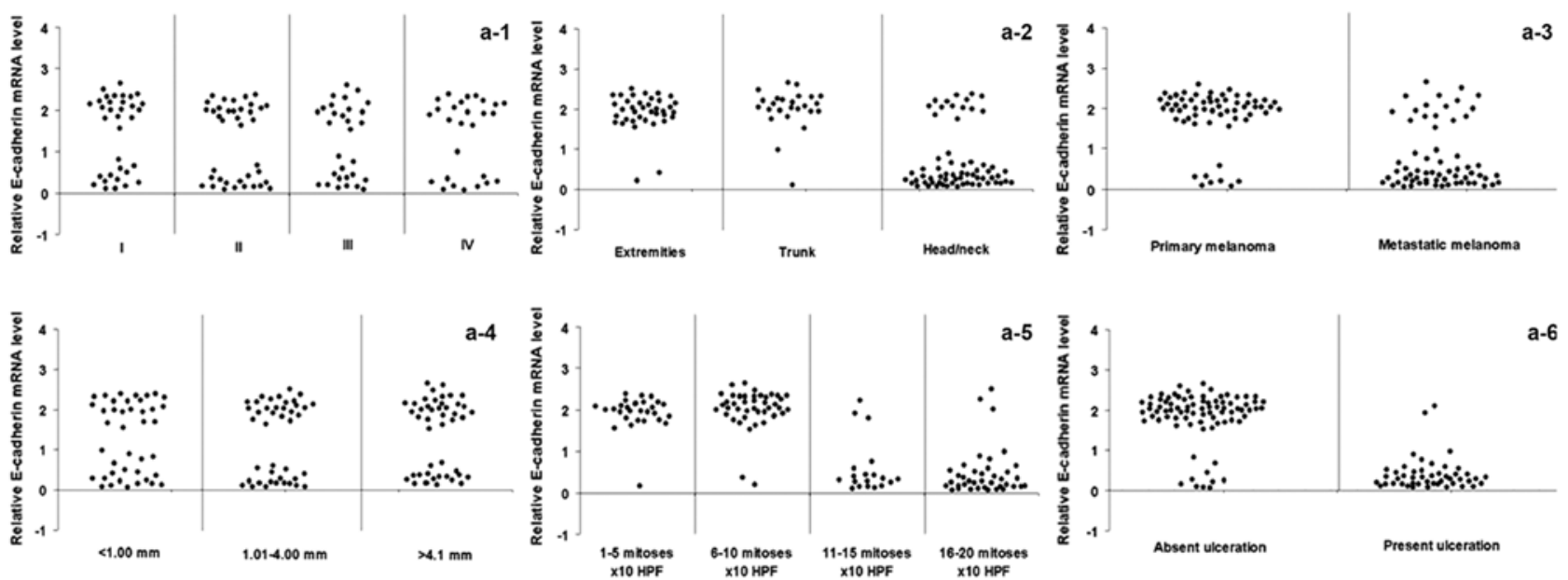

B

MUCOSAL MELANOMA
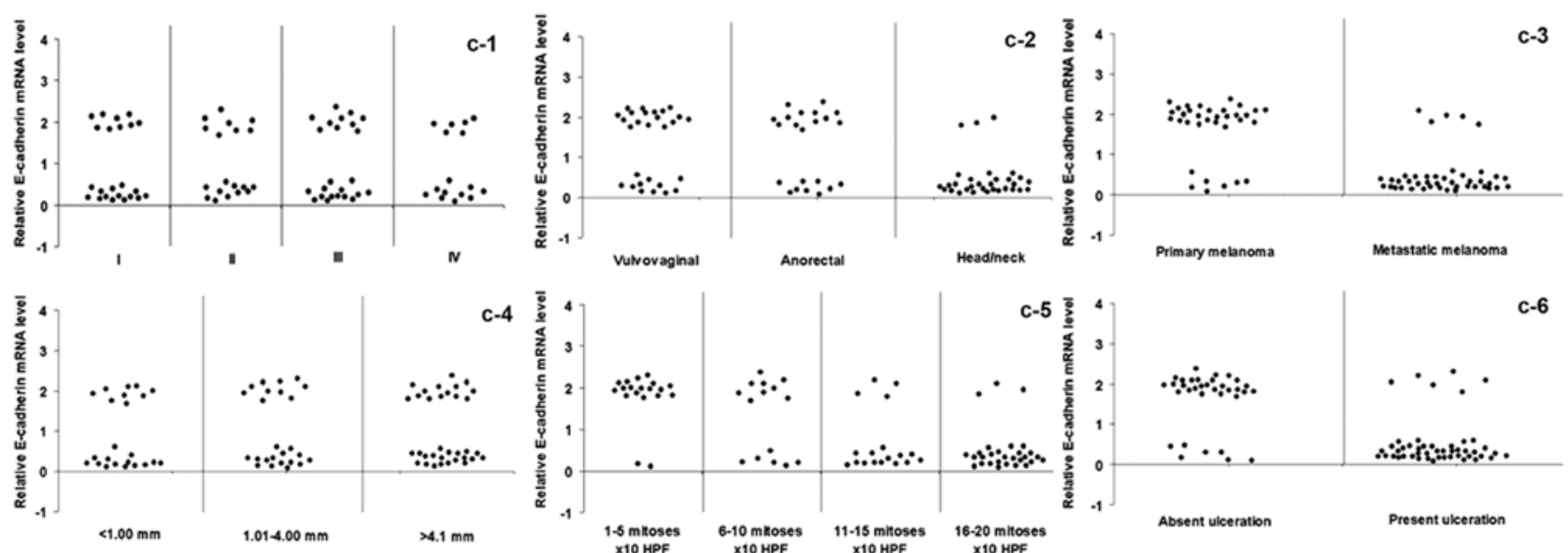

C

UVEAL MELANOMA
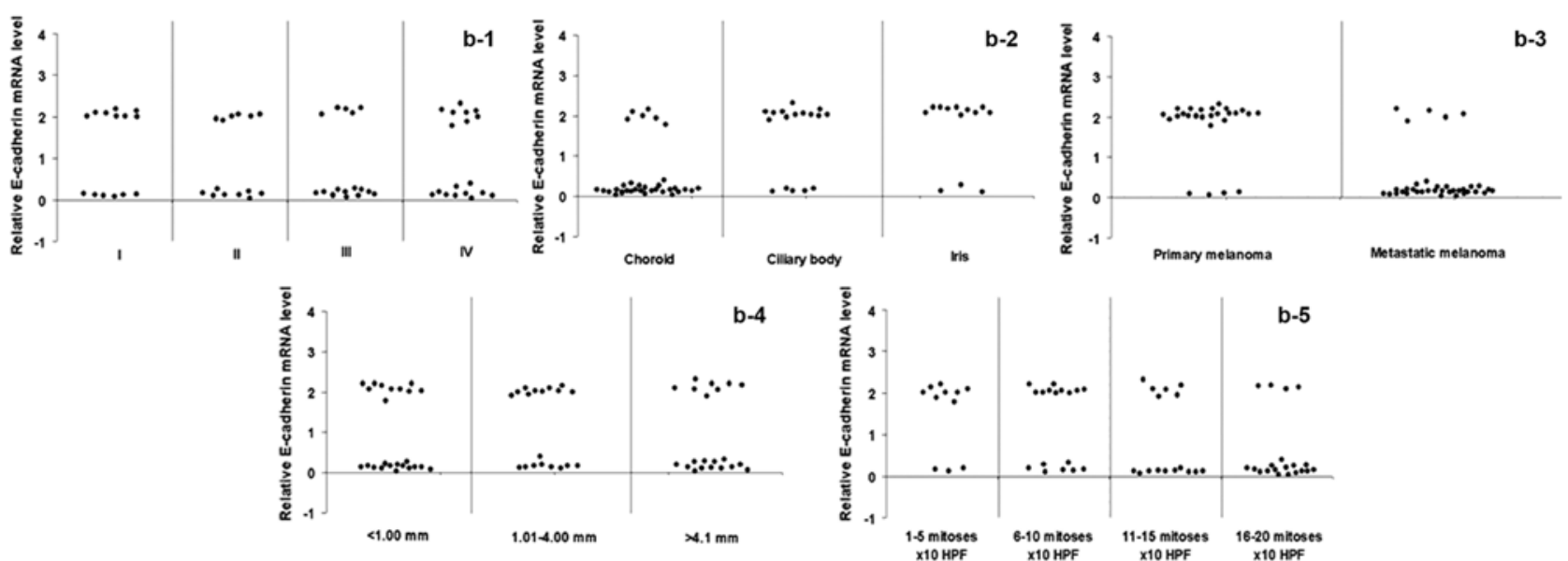

Figure 4. Correlations between E-cadherin expression and clinicopathological characteristics in cutaneous, mucosal and uveal melanoma. In (A) cutaneous and (B) mucosal melanomas, decreased E-cadherin expression was correlated with head/neck localization $\left(\mathrm{p}<0.0001 ; \chi^{2}\right.$ test $)(\mathrm{a}-2$ and $\mathrm{b}-2)$, metastasis $(\mathrm{p}<0.0001$; $\chi^{2}$ test) (a-3 and $\left.b-3\right)$, mitotic count ( $\mathrm{p}<0.0001 ; \chi^{2}$ test) ( -5 and $\left.b-5\right)$ and ulceration ( $<<0.0001 ; \chi^{2}$ test) ( $\mathrm{a}-6$ and $\left.\mathrm{b}-6\right)$. No association was found between E-cadherin expression and AJCC stage (cutaneous melanoma, $\mathrm{p}=0.336$; mucosal melanoma, $\mathrm{p}=0.358 ; \chi^{2}$ test) (a-1 and $\mathrm{b}-1$ ) and Breslow thickness (cutaneous melanoma, $\mathrm{p}=0.161$; mucosal melanoma, $\mathrm{p}=0.207, \chi^{2}$ test) (a-4 and $\mathrm{b}-4$ ). In (C) uveal melanoma, decreased E-cadherin expression was correlated with choroidal localization $\left(\mathrm{p}<0.0001 ; \chi^{2}\right.$ test) $(\mathrm{c}-2)$, metastasis $\left(\mathrm{p}<0.0001 ; \chi^{2}\right.$ test) $(\mathrm{c}-3)$ and mitotic index $\left(\mathrm{p}=0.002 ; \chi^{2}\right.$ test $)(\mathrm{c}-5)$. No association was found between E-cadherin expression and AJCC stage $\left(\mathrm{p}=0.424 ; \chi^{2}\right.$ test $)(\mathrm{c}-1)$ and Breslow thickness $\left(\mathrm{p}=0.385 ; \chi^{2}\right.$ test $)(\mathrm{c}-4)$.

of E-cadherin expression it was: 2 months, 60 months, average 32.7 months, median 28 months in patients with cutaneous melanoma; 2 months, 60 months, average 31.6 months, median 28 months in patients with mucosal melanoma; 2 months 


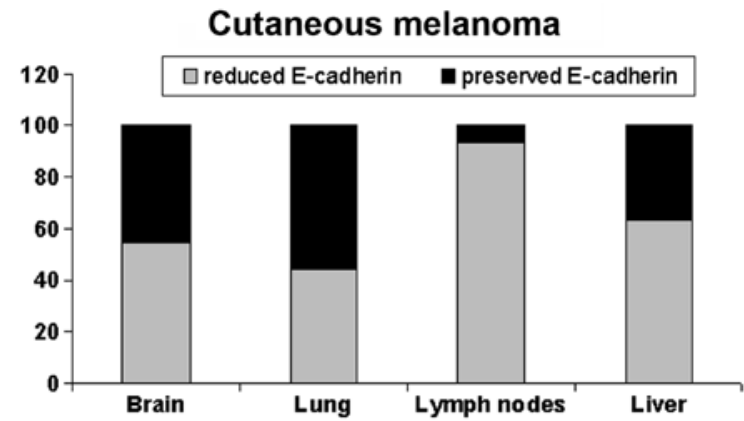

Uveal melanoma

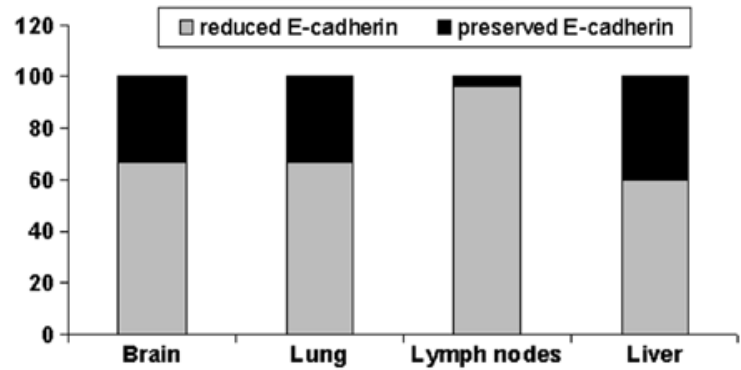

Mucosal melanoma

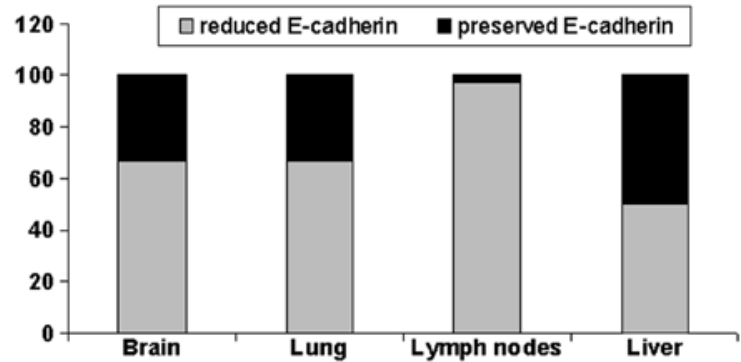

Figure 5. Correlation between reduced E-cadherin expression and organspecific metastasis. Graph shows the organ distribution of metastases. The plot indicates the percentage of cases with reduced vs. preserved E-cadherin mRNA expression levels.

60 months, average 31.9 months, median 32 months in patients with uveal melanoma. In the rest of the patients it was: 2 months, 40 months, average 21.4 months, median 20 months in patients with cutaneous melanoma; 2 months, 56 months, average 21.5 months, median 20 months in patients with mucosal melanoma; 2 months, 36 months, average 16.9 months, median 16 months in patients with uveal melanoma.

\section{Discussion}

The role of E-cadherin as a 'suppressor of invasion' (15) has been well established in the context of melanoma, since its re-expression was accompanied by suppression of tumor invasiveness and metastasis (16). Moreover, the significant role played by E-cadherin in melanoma was very recently evidenced by observations that the resistance to BRAF inhibitors displayed by melanomas harboring mutations in $B R A F$ was strictly associated with a significant decrease in E-cadherin expression (17). However, although reduced E-cadherin expression has been well documented in malignant melanoma and is associated with specific features of the tumor (17-19), to the best of our knowledge, the underlying mechanisms have not been extensively determined. In fact, in only two cellular melanoma models the relationship between $E$-cadherin silencing and promoter methylation was evidenced $(19,20)$. In the present study, we showed that in cutaneous, uveal, and mucosal melanomas the methylation at specific $\mathrm{CpG}$ sites in the $E$-cadherin promoter was responsible for reduced gene expression (Figs. 1 and 2) and several clinicopathological features. Indeed, E-cadherin downregulation was assumed to be an important step in the progression from a melanoma in situ to an invasive and metastasizing lesion and a determinant of tumor depth $(8,21)$. Thus, the demonstration that E-cadherin expression is epigenetically regulated through promoter methylation and that it can be reverted by demethylating tools, such as 5-aza-dC treatment or DNMT1 silencing it may be of clinical significance (Fig. 3). This latter data accounts for the prominent role of DNMT1 in repressing E-cadherin transcription, as already shown in another cancer model (22), and is in agreement with studies reporting the near selective activity of 5-aza-dC in inhibiting DNMT1 and neglecting DNMT3a and DNMT3b enzymes $(23,24)$. The identification of a likely mechanism for E-cadherin downregulation in melanoma may pave the way to alternative therapeutic strategies. This possibility is further suggested by additional results of the present study, which indicate that methylation and reduced expression of E-cadherin are closely associated with clinicopathological markers, either specific or common

\section{$\square$ untreated cells $\square 5$-aza-dC-treated cells}

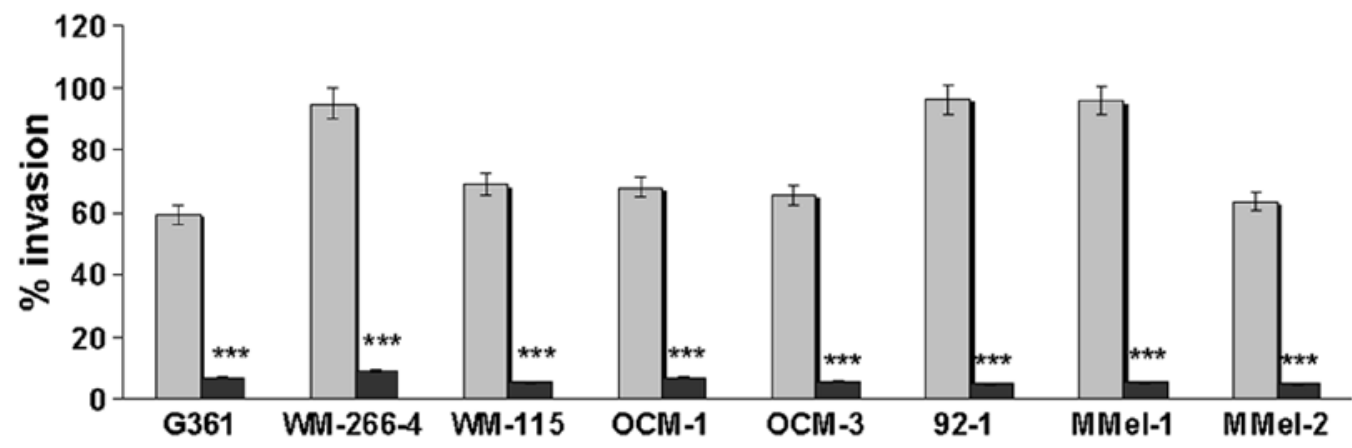

Figure 6. Reduced melanoma invasiveness after 5-aza-dC treatment. Invasive activity was evaluated by the Cultrex ${ }^{\circledR}$ BME cell invasion assay. Experiments were run in triplicate and the data are expressed as the mean \pm SD. Significant ${ }^{* * * *} \mathrm{p}<0.001,5$-aza-dC-treated vs. untreated melanoma cells. 
CUTANEOUS MELANOMA
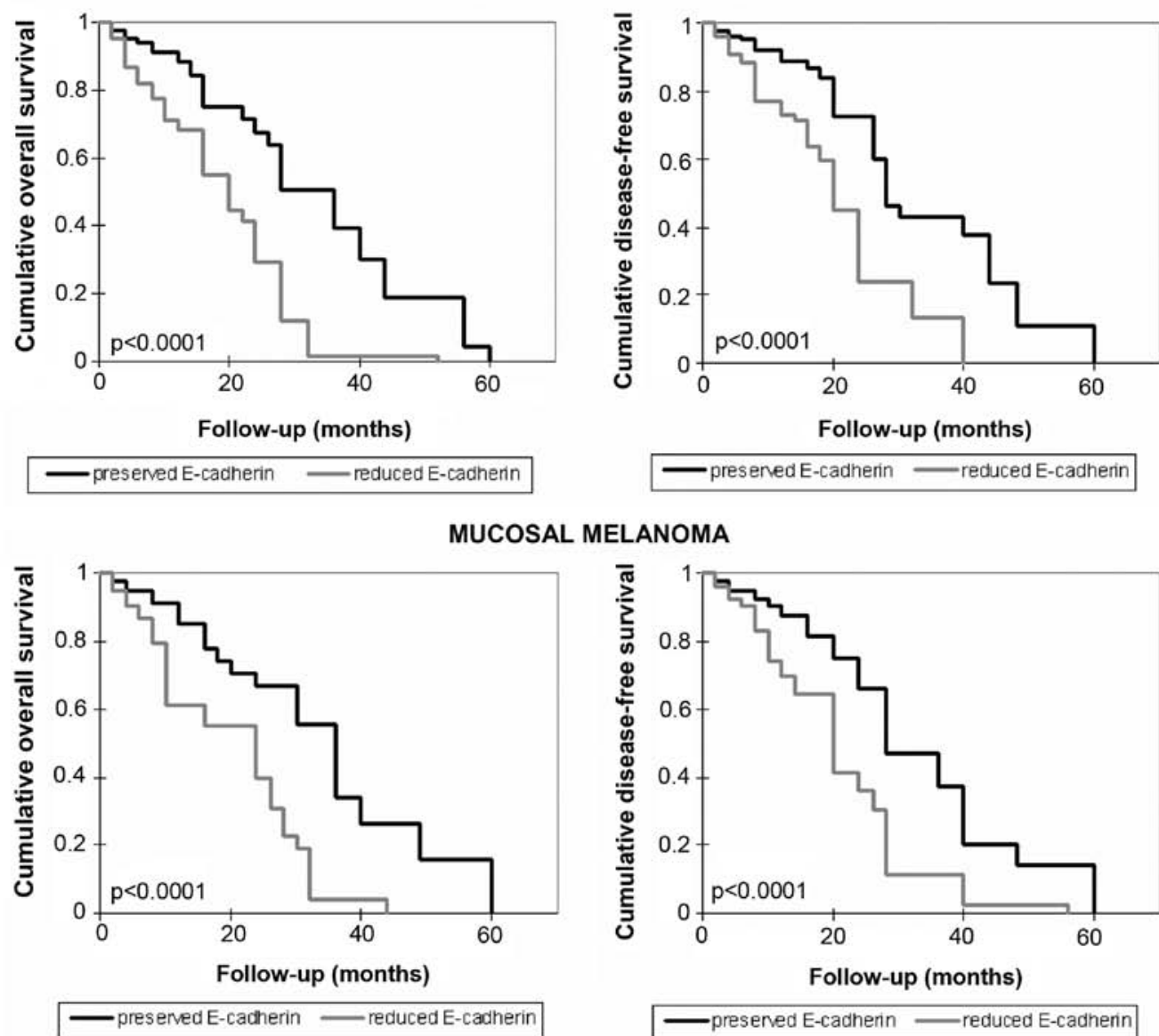

- preserved E-cadherin reduced E-cadherin

- presened E-cadherin —reduced E-cadherin

UVEAL MELANOMA
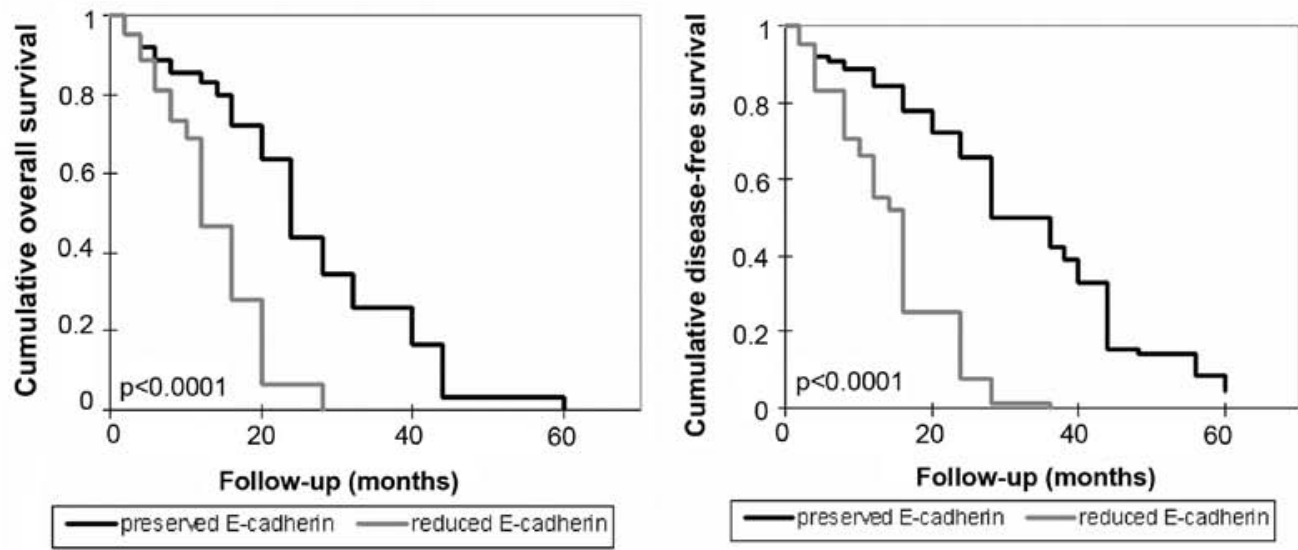

- preserved E-cadherin —reduced E-cadherin

Figure 7. Relationship between E-cadherin content and cumulative overall and disease-free survival in melanoma patients. Kaplan-Meier survival curves relative to patients with high and low E-cadherin expression are shown.

to each type of melanoma. This may be of some interest, since up-to-date conflicting data emerge from the literature. Initially, E-cadherin expression was shown to be rare in both nevocellular nevi and early-stage melanoma, but preserved in advanced-stage melanoma and melanoma metastases (25). More recently, however, E-cadherin expression was found to be altered in different stages of melanoma progression (19). Moreover, information with regard to the actual meaning of $E$-cadherin epigenetic silencing in melanoma was lacking. In the present study, we showed that in cutaneous melanoma reduced E-cadherin expression via promoter aberrant methylation was strongly associated with several poor prognostic factors, namely ulceration, head/neck localization, mitotic count and metastasis as well as with reduced overall/disease-free survival (Figs. 4 and 7). No statistical association was found with clinical stage or Breslow thickness. Our findings allow us to hypothesize that reduced E-cadherin expression through promoter hypermethylation may have a major impact on the proliferation rate of melanoma. The inverse association between E-cadherin expression and the mitotic index found by us is in line with various studies, which reveal that downregulation of E-cadherin prevents the control 
of melanocyte proliferation by keratinocytes and is involved in melanocyte transformation $(16,26)$. Moreover, other studies have reported that expression of E-cadherin results in inhibition of cell motility and local invasion in melanoma cells $(16,27)$, as well as in other cell systems $(28,29)$. The higher frequency of $E$-cadherin methylation and silencing in cutaneous melanoma occurring in head and neck regions, as detected in the present study, deserves to be considered separately. The incidence of melanoma of the head and neck has increased in proportion to sun exposure, and it is often linked to poor prognosis and reduced survival (30). Intense vascularization and abundance of lymphatic vessels in the region may account for these malignancy characteristics, but although it is generally believed that molecular mechanisms different from those involved in melanoma occurring in sun-protected areas are engaged $(31,32)$, little information about distinctive molecular markers of head/neck melanoma are available. The positive correlation between the methylation of E-cadherin and the location of melanoma in the head and neck region highlighted here may explain the apparent discrepancies with other studies reporting a low frequency of methylation of the gene in this tumor. Indeed, in case studies by Liu et al, melanomas of the head and neck were not included (33). The silencing of E-cadherin through aberrant promoter methylation may contribute to add new insights into the mechanisms accounting for the higher malignancy of head/neck melanoma. A similar hypothesis may be advanced for head/neck mucosal melanomas, which, although rare (34), represent half of all mucosal melanomas and exhibit a more aggressive behavior as compared to mucosal melanoma of other regions (35). Indeed, it is quite intriguing that, among the various types of mucosal melanoma, methylation-mediated downregulation of E-cadherin is almost entirely restricted to the head and neck area (Fig. 4). Likewise, we showed that the presence of ulcerated lesions, high mitotic index, metastasis and reduced overall/disease-free survival are conditions frequently associated with epigenetic downregulation of E-cadherin in mucosal melanomas (Figs. 4 and 7). These data allow us to hypothesize that cutaneous and mucosal melanomas not only use the same mechanism for regulating E-cadherin expression, but also that both adopt it in the same circumstances of transformation. Regarding uveal melanoma, it is worth mentioning that the expression of E-cadherin, along with other biomarkers, is considered to be the most accurate predictor of metastasis (36). Therefore, our findings that the downregulation of E-cadherin correlates inversely with the mitotic index and directly with the choroidal site, metastasis and reduced overall/disease-free survival (Figs. 4 and 7) are not surprising, albeit indicative from a clinicopathological point of view. In fact, it is well recognized that high expression of E-cadherin is strictly associated to the epithelial phenotype, while loss of E-cadherin is indicative of mesenchymal transition and acquirement of invasiveness characteristics $(37,38)$. In addition, it is even more interesting that all the melanoma types share the association of methylation-induced E-cadherin reduction with the metastatic phenotype (Fig. 4), the lymph nodal metastasis localization (Fig. 5), the invasive character (Fig. 6), and the reduced overall/disease-free survival (Fig. 7). Such data, streaming from the well known role exerted by E-cadherin as cell-cell adhesion molecule and central hub of inhibitory signals of cell motility $(39,40)$, contribute to clarify why melanoma preferentially metastasizes to distant sites and lymph nodes. Taken together, the results reported in the present study highlight the crucial role of E-cadherin in the events affecting melanoma progression and support the proposal that E-cadherin itself may be considered as a prognostic factor for melanoma.

In conclusion, in the light of the above considerations, the findings reported in the present study, indicate that promoter hypermethylation plays a significant role in switching off E-cadherin gene expression in malignant melanoma and suggest that loss of E-cadherin may contribute to increased cell proliferation, invasiveness, ulcerative process, metastasis, prevalently at lymph nodes, head and neck or choroidal localization, and reduced disease-free/overall survival. Taken together, the findings reported in the present study suggest that E-cadherin downregulation may itself be considered a poor prognostic factor in melanoma and raise the possibility that reactivation of $E$-cadherin through promoter demethylation may represent a promising therapeutic strategy for the treatment of melanoma.

\section{Acknowledgements}

The authors acknowledge the critical review by Professor Giuseppe Teti. The present study was supported in part by funds granted to Scylla Biotech Srl by the Ministero dell'Università e della Ricerca Scientifica of Italy (project n.4/13 ex art. 11 D.M. n. 593).

\section{References}

1. Demierre MF, Sabel MS, Margolin KA, Daud AI and Sondak VK: State of the science 60th anniversary review: 60 Years of advances in cutaneous melanoma epidemiology, diagnosis, and treatment, as reported in the journal Cancer. Cancer 113 (Suppl 7): S1728-S1743, 2008.

2. Venza M, Visalli M, Biondo C, Lentini M, Catalano T, Teti D and Venza I: Epigenetic regulation of $p 14^{A R F}$ and $p 16^{I N K 4 A}$ expression in cutaneous and uveal melanoma. Biochim Biophys Acta 1849: 247-256, 2015.

3. Venza I, Visalli M, Oteri R, Teti D and Venza M: Class I-specific histone deacetylase inhibitor MS-275 overrides TRAILresistance in melanoma cells by downregulating c-FLIP. Int Immunopharmacol 21: 439-446, 2014.

4. Venza M, Visalli M, Catalano T, Fortunato C, Oteri R, Teti D and Venza I: Impact of DNA methyltransferases on the epigenetic regulation of tumor necrosis factor-related apoptosis-inducing ligand (TRAIL) receptor expression in malignant melanoma. Biochem Biophys Res Commun 441: 743-750, 2013.

5. Venza I, Visalli M, Oteri R, Cucinotta M, Teti D and Venza M: Class II-specific histone deacetylase inhibitors MC1568 and MC1575 suppress IL-8 expression in human melanoma cells. Pigment Cell Melanoma Res 26: 193-204, 2013.

6. Venza M, Visalli M, Biondo C, Oteri R, Agliano F, Morabito S, Caruso G, Caffo M, Teti D and Venza I: Epigenetic effects of cadmium in cancer: Focus on melanoma. Curr Genomics 15: 420-435, 2014.

7. Schneider MR and Kolligs FT: E-cadherin's role in development, tissue homeostasis and disease: Insights from mouse models: Tissue-specific inactivation of the adhesion protein E-cadherin in mice reveals its functions in health and disease. BioEssays 37: 294-304, 2015.

8. Andersen K, Nesland JM, Holm R, Flørenes VA, Fodstad $\varnothing$ and Maelandsmo GM: Expression of S100A4 combined with reduced E-cadherin expression predicts patient outcome in malignant melanoma. Mod Pathol 17: 990-997, 2004.

9. Kuphal S and Bosserhoff AK: E-cadherin cell-cell communication in melanogenesis and during development of malignant melanoma. Arch Biochem Biophys 524: 43-47, 2012. 
10. Graff JR, Gabrielson E, Fujii H, Baylin SB and Herman JG: Methylation patterns of the E-cadherin 5' CpG island are unstable and reflect the dynamic, heterogeneous loss of E-cadherin expression during metastatic progression. J Biol Chem 275: 2727-2732, 2000.

11. Venza M, Visalli M, Biondo C, Oteri R, Agliano F, Morabito S, Teti D and Venza I: Epigenetic marks responsible for cadmiuminduced melanoma cell overgrowth. Toxicol In Vitro 29: 242-250, 2015.

12. Venza M, Visalli M, Oteri R, Agliano F, Morabito S, Teti D and Venza I: The overriding of TRAIL resistance by the histone deacetylase inhibitor MS-275 involves c-myc up-regulation in cutaneous, uveal, and mucosal melanoma. Int Immunopharmacol 28: 313-321, 2015.

13. Herman JG, Graff JR, Myöhänen S, Nelkin BD and Baylin SB: Methylation-specific PCR: A novel PCR assay for methylation status of CpG islands. Proc Natl Acad Sci USA 93: 9821-9826, 1996.

14. Ribeiro-Filho LA, Franks J, Sasaki M, Shiina H, Li LC, Nojima D, Arap S, Carroll P, Enokida H, Nakagawa M, et al: $\mathrm{CpG}$ hypermethylation of promoter region and inactivation of E-cadherin gene in human bladder cancer. Mol Carcinog 34: $187-198,2002$

15. Vleminckx K, Vakaet L Jr, Mareel M, Fiers W and van Roy F: Genetic manipulation of E-cadherin expression by epithelial tumor cells reveals an invasion suppressor role. Cell 66: 107-119, 1991.

16. Hsu MY, Meier FE, Nesbit M, Hsu JY, Van Belle P, Elder DE and Herlyn M: E-cadherin expression in melanoma cells restores keratinocyte-mediated growth control and down-regulates expression of invasion-related adhesion receptors. Am J Pathol 156: 1515-1525, 2000

17. Liu S, Tetzlaff MT, Wang T, Yang R, Xie L, Zhang G, Krepler C, Xiao M, Beqiri M, Xu W, et al: $\mathrm{miR}-200 \mathrm{c} / \mathrm{Bmil}$ axis and epithelial-mesenchymal transition contribute to acquired resistance to BRAF inhibitor treatment. Pigment Cell Melanoma Res 28: 431-441, 2015.

18. Lade-Keller J, Riber-Hansen R, Guldberg P, Schmidt H, Hamilton-Dutoit SJ and Steiniche T: E- to N-cadherin switch in melanoma is associated with decreased expression of phosphatase and tensin homolog and cancer progression. Br J Dermatol 169: 618-628, 2013.

19. Seleit IA, Samaka RM, Basha MA and Bakry OA: Impact of E-cadherin expression pattern in melanocytic nevi and cutaneous malignant melanoma. Anal Quant Cytopathol Histpathol 34: 204-213, 2012.

20. Tsutsumida A, Hamada J, Tada M, Aoyama T, Furuuchi K, Kawai Y, Yamamoto Y, Sugihara T and Moriuchi T: Epigenetic silencing of $\mathrm{E}$ - and $\mathrm{P}$-cadherin gene expression in human melanoma cell lines. Int J Oncol 25: 1415-1421, 2004.

21. Johnson JP: Cell adhesion molecules in the development and progression of malignant melanoma. Cancer Metastasis Rev 18: 345-357, 1999.

22. Qiu X, Qiao F, Su X, Zhao Z and Fan H: Epigenetic activation of E-cadherin is a candidate therapeutic target in human hepatocellular carcinoma. Exp Ther Med 1: 519-523, 2010.

23. Ghoshal K, Datta J, Majumder S, Bai S, Kutay H, Motiwala T and Jacob ST: 5-Aza-deoxycytidine induces selective degradation of DNA methyltransferase 1 by a proteasomal pathway that requires the KEN box, bromo-adjacent homology domain, and nuclear localization signal. Mol Cell Biol 25: 4727-4741, 2005.

24. Palii SS, Van Emburgh BO, Sankpal UT, Brown KD and Robertson KD: DNA methylation inhibitor 5-Aza-2'deoxycytidine induces reversible genome-wide DNA damage that is distinctly influenced by DNA methyltransferases 1 and $3 \mathrm{~B}$. Mol Cell Biol 28: 752-771, 2008.
25. Danen EHJ, de Vries TJ, Morandini R, Ghanem GG, Ruiter DJ and van Muijen GNP: E-cadherin expression in human melanoma. Melanoma Res 6: 127-131, 1996.

26. Hsu M, Andl T, Li G, Meinkoth JL and Herlyn M: Cadherin repertoire determines partner-specific gap junctional communication during melanoma progression. J Cell Sci 113: 1535-1542, 2000.

27. Lu Z, Ghosh S, Wang Z and Hunter T: Downregulation of caveolin-1 function by EGF leads to the loss of E-cadherin, increased transcriptional activity of beta-catenin, and enhanced tumor cell invasion. Cancer Cell 4: 499-515, 2003.

28. Zhang L, Wang G, Wang L, Song C, Wang X and Kang J: Valproic acid inhibits prostate cancer cell migration by up-regulating E-cadherin expression. Pharmazie 66: 614-618, 2011.

29. Bocca C, Bozzo F, Francica S, Colombatto S and Miglietta A: Involvement of PPAR gamma and E-cadherin/beta-catenin pathway in the antiproliferative effect of conjugated linoleic acid in MCF-7 cells. Int J Cancer 121: 248-256, 2007.

30. Larson DL and Larson JD: Head and neck melanoma. Clin Plast Surg 37: 73-77, 2010.

31. Whiteman DC, Watt P, Purdie DM, Hughes MC, Hayward NK and Green AC: Melanocytic nevi, solar keratoses, and divergent pathways to cutaneous melanoma. J Natl Cancer Inst 95: 806-812, 2003.

32. Maldonado JL, Fridlyand J, Patel H, Jain AN, Busam K, Kageshita T, Ono T, Albertson DG, Pinkel D and Bastian BC: Determinants of BRAF mutations in primary melanomas. J Natl Cancer Inst 95: 1878-1890, 2003.

33. Liu S, Ren S, Howell P, Fodstad O and Riker AI: Identification of novel epigenetically modified genes in human melanoma via promoter methylation gene profiling. Pigment Cell Melanoma Res 21: 545-558, 2008.

34. Bakkal FK, Başman A, Kızıl Y, Ekinci Ö, Gümüşok M, Ekrem Zorlu M and Aydil U: Mucosal melanoma of the head and neck: Recurrence characteristics and survival outcomes. Oral Surg Oral Med Oral Pathol Oral Radiol 120: 575-580, 2015.

35. Gavriel H, McArthur G, Sizeland A and Henderson M: Review: Mucosal melanoma of the head and neck. Melanoma Res 21: 257-266, 2011.

36. Chang SH, Worley LA, Onken MD and Harbour JW: Prognostic biomarkers in uveal melanoma: Evidence for a stem cell-like phenotype associated with metastasis. Melanoma Res 18: 191-200, 2008.

37. Tucci MG, Lucarini G, Brancorsini D, Zizzi A, Pugnaloni A, Giacchetti A, Ricotti $G$ and Biagini $G$ : Involvement of E-cadherin, beta-catenin, Cdc42 and CXCR4 in the progression and prognosis of cutaneous melanoma. Br J Dermatol 157: 1212-1216, 2007.

38. Zeisberg M and Neilson EG: Biomarkers for epithelial-mesenchymal transitions. J Clin Invest 119: 1429-1437, 2009.

39. Li Q and Mattingly RR: Restoration of E-cadherin cell-cell junctions requires both expression of E-cadherin and suppression of ERK MAP kinase activation in Ras-transformed breast epithelial cells. Neoplasia 10: 1444-1458, 2008.

40. Siret C, Terciolo C, Dobric A, Habib MC, Germain S, Bonnier R, Lombardo D, Rigot V and André F: Interplay between cadherins and $\alpha 2 \beta 1$ integrin differentially regulates melanoma cell invasion. Br J Cancer 113: 1445-1453, 2015. 